\title{
Long-term mortality rate in rheumatoid arthritis patients with disease
}

onset in the 1980s

\section{Kapetanovic, E Lindqvist, P Geborek, T Saxne, K Eberhard}

Department of Rheumatology, Lund University, Lund, Sweden

Meliha C Kapetanovic, Department of Rheumatology, Lund University Hospital, Kioskgatan 3, SE-221 85 Lund, Sweden.

E-mail: meliha.c_kapetanovic@med.lu.se

Running heads:

Right: Long-term mortality in RA

Left: MC Kapetanovic et al

Accepted 16 March 2011 
Objective: To investigate the mortality rate and possible early predictive factors of mortality after 19-23 years in a cohort of patients with rheumatoid arthritis (RA) followed prospectively from disease onset.

Patients and methods: A community-based cohort of 183 patients (63\% female) with RA and disease duration $<2$ years was recruited 1985-1989. The patients were followed yearly from diagnosis until death or 31 December 2008. Mean age and mean duration of symptoms (range) at diagnosis were 52 (18-78) years and 11 (0-24) months, respectively. Death certificates were obtained from the Swedish Cause of Death Register and causes of death coded by the International Classification of Diseases (ICD-10). Death rates of RA patients were compared to those of age- and sex-matched controls. Possible predictors of mortality were analysed using a Cox regression model.

Results: By 31 December 2008, 69 patients (37 women and $32 \mathrm{men}$ ) had died. The standardized mortality ratio (SMR) was 1.23 [95\% confidence interval (CI) 0.97-1.55] and p $<0.09$. Older age, male sex, smoking, and the presence of cardiovascular disease (CVD) at RA diagnosis were identified as early predictors of mortality. CVD was the most common cause of death (46\%), followed by malignancies $(29 \%)$ and infections (13\%). RA was not stated as the direct cause of death in any patient and was mentioned among underlying causes in only $16 / 69(23 \%)$ patients.

Conclusion: Mortality rate after 19-23 years of disease duration in this cohort of patients with disease onset in the 1980s was not significantly increased compared to age- and sexmatched controls. No RA disease-related factor predicted mortality. 
Rheumatoid arthritis (RA) is a chronic inflammatory disease commonly associated with joint destruction, disability, and increased co-morbidity. The issue of premature mortality in patients with RA has been addressed in numerous studies. The first study reporting an increased mortality in patients with RA compared to controls was published in 1953 (1), and was followed by several reports confirming these findings (2-9). In recent decades the approach to treatment of RA and treatment strategies in RA has changed dramatically. Early treatment with disease-modifying anti-rheumatic drugs (DMARDs) and the availability of biological remedies have led to better control of disease activity and decreased disability (1014). However, in a population-based analysis of trends over four decades published in 2003 , an increased mortality rate [standardized mortality ratio (SMR) of 1.27] in RA patients was found (6). Overall mortality in the general population was shown to decrease over 4-5 decades but mortality rates in RA remained relatively constant, resulting in a widening of the mortality gap (7). A recently published review of $>200$ studies on mortality in RA reported lower SMRs in studies of inception cohorts compared to non-inception cohorts over 60 years (median SMRs of 1.2-1.3 and 1.6-1.7, respectively) (15).

Krause et al (16) found that a sufficient response to methotrexate (MTX) treatment was associated with decreased mortality in patients with severe RA, although the mortality rate remained only modestly increased compared to that in patients with severe RA not responding to MTX (SMR 1.64 vs. 4.11, respectively). MTX has also been shown to provide a survival benefit largely by reducing cardiovascular mortality (17). Similarly, women but not men with RA treated with tumour necrosis factor (TNF) inhibitors showed reduced mortality compared to those treated with conventional DMARDs (14).

Studies reporting no increased mortality rate mostly have fairly short follow-up times. In our previously published results, including patients from the present cohort, the mortality rate was neither increased after 8-13 nor after 13-18 years (SMR 0.87 and 1.33, 
respectively) compared to age- and sex-matched controls $(18,19)$. No excess mortality was found in another study where actively treated RA patients were followed up for 7-14 years after diagnosis (20). A recently published study including all incident cases of RA with disease onset in the 2000s in Finland reported no increased risk of death (21). In a Dutch inception cohort study including $>600$ early RA patients, there was no increased mortality during the first 10 years (22) but thereafter the mortality rate increased during the years 11-23 of follow-up (23).

Possible predictors of excess mortality have been examined in several studies. High disease activity at the onset was found to be associated with increased mortality in some reports $(3,20,24)$. Other reported risk factors for increased mortality are the presence of extra-articular features $(3,17)$, high age, male sex, rheumatoid factor positivity $(3,5,23)$, and functional status $(2,3)$.

The aim of the present study was to assess whether a low mortality rate was maintained in our cohort of early RA patients that have now been followed from diagnosis up to $19-23$ years. We also aimed to identify possible early predictors of mortality.

\section{Patients and methods}

The study population comprised a cohort of 183 patients with early RA followed prospectively from diagnosis until death or 31 December 2008. The inclusion in the study took place between 1985 and 1989. The vast majority of patients were referred from the primary care in the recruitment area for Lund University Hospital. Patients older than 18 years and fulfilling 1958 American Rheumatism Association (ARA) classification criteria for definitive RA were eligible for the study and were included at the time of diagnosis (25). Baseline characteristics, laboratory and clinical assessment, and follow-up details have been 
described previously $(26,27)$. In brief, $115(63 \%)$ women and $68(43 \%)$ men participated in the study. Mean (SD) age and duration of symptoms at inclusion were 52 (12) years and 11 (7) months, respectively. Mean (SD) follow-up time for the whole cohort at 31 December 2008 was 18 (5) years. The patients were evaluated annually with standardized clinical and laboratory measures. Disease activity measures included the Ritchie articular index, a 44-joint count of swollen and tender joints, patient's visual analogue scale (VAS $100 \mathrm{~mm}$ ) for global health, and erythrocyte sedimentation rate (ESR), thus permitting the calculation of the original Disease Activity Score (DAS) (28). Activity limitations were evaluated using the validated Swedish version of the Health Assessment Questionnaire (HAQ) (29). Radiographs of hands and feet were taken yearly for the first 5 years and thereafter every 5 years and scored by the Larsen method (30). The presence of shared epitopes was analysed using polymerase chain reaction (PCR), and rheumatoid factors and anti-cyclic citrullinated peptide (anti-CCP)-2 antibodies were analysed with an enzyme-linked immunosorbent assay (ELISA) using commercial kits as described previously (31). Standardized information on general medical history, including previous and coexisting diseases, concomitant medications, and also smoking habits, was collected at inclusion.

Throughout the study all patients with active disease were offered treatment with DMARDs according to current clinical practice. About $50 \%$ of patients started treatment with DMARDs within a year of diagnosis. D-Penicillamine and antimalarials were most commonly used in the early years and MTX became most frequently used during the 1990s. In total, $145(79 \%)$ patients were exposed to different DMARDs over the study period, including 95 patients (52\%) who received MTX. A total of 28 patients (15\%) in this cohort were treated with biological remedies, mostly TNF antagonists, for at least some period of time. 
Information on vital status was obtained from Statistics Sweden (the Swedish Cause of Death Register) up to 31 December 2008 (32). SMRs were computed using these national Swedish mortality and census data for the same time period, adjusting for calendar year, age, and sex.

Approval from the Ethical Review Board at Lund University (LU 525-02) was obtained for this study along with informed consent from each patient.

Statistical analysis

Distribution of continuous variables was calculated as mean (min-max; SD). Differences between groups were analysed using the $\chi^{2}$ test and the Mann-Whitney U-test when appropriate. Death certificates were obtained from the National Board of Health and Welfare in Sweden (the Swedish Cause of Death Register) and causes of death coded by the International Classification of Diseases (ICD-10); these were compared to age- and sexmatched controls from both the whole country and the same geographical area (Skania region). SMRs with 95\% confidence interval (CI) and p-values (two-sided) were calculated according to www.openepi.com. To study possible baseline predictors of mortality, Cox regression models were created.

\section{Results}

By 31 December 2008, a total of 69 (38\%) patients (37 women and 32 men) in this cohort had died. Mean (SD) follow-up time until death was 13 (5) years. Baseline demographic and disease characteristics for the whole cohort, the patients who died, and those still living are given in Table 1. 
The SMR calculated after 19-23 years of follow-up was 1.23 (95\% CI $0.97-$ 1.55). The mortality rate in this cohort tended to be higher than that of age- and sex-matched controls from the whole of Sweden but the difference was not significant $(p<0.09)$. The SMR for men $(1.55,95 \%$ CI 1.06-2.19) was higher than for women and the results were statistical significant (SMR 1.04, 95\% CI 0.74-1.44). SMRs were similar for RF-positive and RF-negative patients (1.26, 95\% CI 0.93-1.66 and 0.98, 0.55-1.62, respectively). Moreover, there were no statistically significant differences when mortality in this cohort was compared to that of age- and sex-matched controls from the same geographical area.

Figure 1 shows the number of observed deaths and 95\% CIs compared to the number of expected deaths for age- and sex-matched controls from Sweden.

Death certificates were available in 64 of the 69 patients. For the remaining five patients, information on cause of death was obtained from the medical records. Cardiovascular disease (CVD) was the most common cause of death $(32 / 69 ; 46 \%)$, followed by malignancy $(20 / 69 ; 29 \%)$, severe infection $(9 / 69 ; 13 \%)$, respiratory diseases $(3 / 69 ; 4 \%)$, and other courses $(5 / 69 ; 7 \%)$ including alcohol intoxication $(n=1)$, uraemia $(n=1)$, advanced age, and general weakness $(n=3)$. CVD included: angina pectoris, myocardial infarction, transient ischaemic attack (TIA/stroke), congestive heart disease, and peripheral atherosclerotic arterial disease. Malignancies were: lung carcinoma $(n=5)$, haematological malignancies $(n=4)$, carcinoma of the brain $(n=2)$, gastrointestinal malignancies $(n=2)$, breast $(\mathrm{n}=1)$, liver $(\mathrm{n}=1)$, kidney $(\mathrm{n}=1)$, tonsil $(\mathrm{n}=1)$, and cervical carcinoma $(\mathrm{n}=1)$, and malignancies with an unknown primary tumour $(n=2)$. RA was not stated as the direct cause of death in any patient but was mentioned among underlying causes in 16/69 (23\%) patients.

To study possible early predictors of mortality, a Cox regression model was created (Table 2). Higher age at RA diagnosis, male sex, smoking, and the presence of concomitant diseases, in particular CVD, were found to be associated with increased 
mortality. Patients who died had a higher Larsen score at RA diagnosis compared to other patients in the cohort $(\mathrm{p}=0.003$, Table 1$)$, but having more advanced baseline radiographic changes was not predictive for future mortality after adjustment for other baseline characteristics in the regression analysis.

Activity limitation measured by the HAQ or the DAS was not associated with increased mortality. The majority of patients had already been treated with non-steroidal antiinflammatory drugs (NSAIDs) at RA diagnosis but these remedies were not found to have a negative impact on subsequent mortality. Only a few patients had received steroids at RA diagnosis and further analysis was not performed.

To analyse whether the degree of inflammation at diagnosis, activity limitation, use of DMARDs and steroids during the first year after diagnosis influenced the mortality rate, we created a second Cox regression model. Eighty-six (47\%) of the patients started any DMARD treatment and $16(9 \%)$ were treated with steroids orally during this period. Treatment with DMARDs or steroids within a year or at year 1 after diagnosis had no impact on the mortality rate in this cohort (Table 3). Similarly, the amount of inflammation, measured as the area under the curve of ESR, or activity limitation, measured as the area under the curve of the HAQ, during the first year after diagnosis did not influence this risk [p $=0.538$; hazard ratio $(\mathrm{HR}) 1.004,95 \%$ CI $0.99-1.02$; and $\mathrm{p}=0.129$; HR 0.648, 95\% CI $0.37-$ 1.13 , respectively].

\section{Discussion}

The overall mortality rate after 19-23 years of disease duration in this cohort of patients with disease onset in the 1980 s was not significantly increased compared to age- and sex-matched controls from either the whole of Sweden or the Skania region. However, compared to 
previous analyses of mortality in this cohort, the trend has changed from an SMR of 0.87 at 8-13 years after inclusion in the study (18), but remains unchanged compared to the SMR after 13-18 years (19). Mortality among men has increased somewhat compared to women (SMR 1.55) in this cohort. Our findings are in contrast to an early RA study from The Netherlands that reported no increased mortality after 10 years (22) but found excess mortality (SMR 1.40, 95\% CI 1.09-1.77) after 10-23 years (23). The number of individuals included in these studies is considerably larger compared to our study, which might have had an impact on the results.

The most common causes of death in this cohort were CVD and malignancies, which is not different from age- and sex-matched controls in the general population in Sweden (32).

Higher age, male gender, smoking, and the presence of concomitant CVD at the time of RA diagnosis were found to be early predictors of mortality. However, baseline and early activity limitation, disease activity level, and medication during the first year were not related to mortality. Of note, despite the high co-morbidity burden, with approximately $43 \%$ patients having co-morbid diseases at RA diagnosis and $>80 \%$ developing additional diseases within 20 years (33), the long-term mortality rate in the present cohort did not differ from ageand sex-matched controls in the general population. These results are intriguing and several explanations are possible.

The current recommendation on early referral of patients with arthritis to rheumatology units in the geographical area is well recognized by primary health-care providers in Sweden (34). Although the recruitment for the present study took place in the mid-1980s that is before these early referral recommendations it was an active campaign directed to all primary care units in the recruitment area for Lund University Hospital. All consecutive patients from primary care units fulfilling the entry criteria were included, and the 
study can thus be considered community based. The inclusion protocol did not exclude patients with particularly serious co-morbidity at RA disease onset. However, such patients may not have been referred to the study and were therefore excluded. This might explain the low SMR in the present cohort compared to results from several other studies $(2-9,15)$. However, such possible inclusion bias might also be present in other settings.

The patients participating in this study were evaluated regularly by a rheumatology team including assessments of a physician, physiotherapist, occupational therapist, and social worker, and recording of standardized clinical and laboratory measures (27). Furthermore, a close collaboration with the Department of Orthopaedic Surgery has been maintained during the study period and the patients were assessed regarding their need of surgery. Rehabilitation of patients with RA was also provided by our department. The patients included in this study were also followed up at a university rheumatology unit with highly specialized health-care facilities.

The increased knowledge on co-morbidity in RA has made rheumatologists more aware of risk factors for CVD, certain malignancies, infections or other diseases, resulting in better preventive efforts and earlier referral to specialized health-care units. Taken together, this suggests that specialized care for the treatment and rehabilitation of patients with RA and access to modern health-care service has a positive impact on long-term mortality.

The improved cardiovascular prevention and treatments have been introduced during the past decade (35), but it is uncertain whether RA patients would benefit more from these than the population in general.

A trend of decreasing mortality in some other chronic diseases such as diabetes mellitus has also been reported. A recent Swedish study examining the mortality of patients with diabetes mellitus over 33 years showed that the excess mortality risk in their patients was 
smaller than previously reported (36). It has also been suggested that RA may be changing into a milder disease (37).

We have reported a lack of association between inflammation and cardiovascular disease in the present cohort (33). Similarly, the amount of inflammation measured by the DAS at RA diagnosis and the area under the curve of DAS within the first year after diagnosis was not found to be predictive for long-term mortality. After an initial peak, the levels of inflammation for the whole cohort remained fairly low over the study period, which might explain why our results diverge from other studies $(3,14,18,24)$. However, ESR at RA diagnosis did not have an impact on the excess of death in a Dutch study either (22).

It has been postulated that a more aggressive treatment strategy with DMARDs early in the course of the disease might have a positive impact on mortality. However, this statement has been questioned and the diverging results are thought to be due to different study designs (15). Treatment with MTX was found to reduce mortality in patients with RA $(16,17)$. As MTX was not commonly used during the first 5-10 years of the present study, we could not perform a corresponding analysis. In total, $47 \%$ of patients in this study started treatment with non-MTX DMARDs within a year of diagnosis. This early DMARD treatment did not influence the long-term mortality in our study, which is in accordance with previously published data (17).

A limitation of the study is the small patient sample and the fact that changes in SMR may have been significant over time with a larger patient group. The trend was, however, unchanged between the two examinations, at 13-18 years and the present one (SMRs of 1.33 and 1.23 , respectively).

As inclusion of patients in this cohort started in the 1980s, ARA 1958 classification criteria for RA were used. These criteria seem to be more sensitive than the 
1987 American College of Rheumatology (ACR) criteria in early studies early (38). When the 1987 ACR criteria were applied to this cohort, all patients fulfilled the classification criteria for RA.

In conclusion, in this community-based early RA cohort, long-term mortality rate was not increased compared to age- and sex-matched controls in the general population. Our results suggest, but by no means prove, that major advances in treatments, more aggressive approaches to treatment, and improved health care for patients with RA during recent decades have contributed to better survival.

\section{Acknowledgements}

This study was supported by grants from the Swedish Rheumatism Association, the Swedish Research Council, the Medical Faculty of the University of Lund, Alfred Österlund's Foundation, the Crafoord Foundation, Greta and Johan Kock's Foundation, the King Gustaf V Foundation, and Lund University Hospital.

\section{References}

1. Cobbs S, Anderson F, Bauer W. Length of life and cause of death in rheumatoid arthritis. N Engl J Med 1953;249:553-6.

2. Allebeck P, Ahlbom A, Allander E. Increased mortality among persons with rheumatoid arthritis, but where RA does not appear on death certificate. Eleven-year follow-up of an epidemiological study. Scand J Rheumatol 1981;10:301-6.

3. Wolfe F, Mitchell DM, Sibley JT, Fries JF, Bloch DA, Williams CA, et al. The mortality of rheumatoid arthritis. Arthritis Rheum 1994;37:481-94. 
4. Symmons DP, Jones MA, Scott DL, Prior P. Longterm mortality outcome in patients with rheumatoid arthritis: early presenters continue to do well. J Rheumatol 1998;25:1072-7.

5. Wallberg-Jonsson S, Ohman ML, Dahlqvist SR. Cardiovascular morbidity and mortality in patients with seropositive rheumatoid arthritis in Northern Sweden. J Rheumatol 1997;24:445-51.

6. Gabriel SE, Crowson CS, Kremers HM, Doran MF, Turesson C, O'Fallon WM, et al. Survival in rheumatoid arthritis: a population-based analysis of trends over 40 years. Arthritis Rheum 2003;48:54-8.

7. Gonzalez A, Maradit Kremers H, Crowson CS, Nicola PJ, Davis JM 3rd, Therneau $\mathrm{TM}$, et al. The widening mortality gap between rheumatoid arthritis patients and the general population. Arthritis Rheum 2007;56:3583-7.

8. Gabriel SE, Michaud K. Epidemiological studies in incidence, prevalence, mortality, and comorbidity of the rheumatic diseases. Arthritis Res Ther 2009;11:229.

9. Nakajima A, Inoue E, Tanaka E, Singh G, Sato E, Hoshi D, et al. Mortality and cause of death in Japanese patients with rheumatoid arthritis based on a large observational cohort, IORRA. Scand J Rheumatol 2010;39:360-7.

10. Maini RN, Breedveld FC, Kalden JR, Smolen JS, Furst D, Weisman MH, et al. Sustained improvement over two years in physical function, structural damage, and signs and symptoms among patients with rheumatoid arthritis treated with infliximab and methotrexate. Arthritis Rheum 2004;50:1051-65.

11. Barra L, Pope JE, Payne M. Real-world anti-tumor necrosis factor treatment in rheumatoid arthritis, psoriatic arthritis, and ankylosing spondylitis: cost-effectiveness based on number needed to treat to improve health assessment questionnaire. $\mathrm{J}$ Rheumatol 2009;36:1421-8. 
12. Hyrich KL, Deighton C, Watson KD; BSRBR Control Centre Consortium, Symmons DP, Lunt M; British Society for Rheumatology Biologics Register. Benefit of antiTNF therapy in rheumatoid arthritis patients with moderate disease activity. Rheumatology (Oxford) 2009;48:1323-7.

13. Augustsson J, Neovius M, Cullinane-Carli C, Eksborg S, van Vollenhoven RF. Patients with rheumatoid arthritis treated with tumour necrosis factor antagonists increase their participation in the workforce: potential for significant long-term indirect cost gains (data from a population-based registry). Ann Rheum Dis 2010;69:126-31.

14. Jacobsson LT, Turesson C, Nilsson JA, Petersson IF, Lindqvist E, Saxne T, et al. Treatment with TNF blockers and mortality risk in patients with rheumatoid arthritis. Ann Rheum Dis 2007;66:670-5.

15. Sokka T, Abelson B, Pincus T. Mortality in rheumatoid arthritis: 2008 update. Clin Exp Rheumatol 2008;26:S35-61.

16. Krause D, Schleusser B, Herborn G, Rau R. Response to methotrexate treatment is associated with reduced mortality in patients with severe rheumatoid arthritis. Arthritis Rheum 2000;43:14-21.

17. Choi HK, Hernán MA, Seeger JD, Robins JM, Wolfe F. Methotrexate and mortality in patients with rheumatoid arthritis: a prospective study. Lancet 2002;359:1173-7.

18. Lindqvist E, Eberhardt K. Mortality in rheumatoid arthritis patients with disease onset in the 1980s. Ann Rheum Dis 1999;58:11-14.

19. Lindqvist E, Geborek P, Saxne T, Nilsson JA, Eberhardt K. Mortality in rheumatoid arthritis patients with disease onset in the 1980s. Arthritis Rheum 2004;50(Suppl);S389. 
20. Peltomaa R, Paimela L, Kautiainen H, Leirisalo-Repo M. Mortality in patients with rheumatoid arthritis treated actively from the time of diagnosis. Ann Rheum Dis 2002;61:889-94.

21. Puolakka K, Kautiainen H, Pohjolainen T, Virta L. No increased mortality in incident cases of rheumatoid arthritis during the new millennium. Ann Rheum Dis 2010;69:2057-8.

22. Kroot EJ, van Leeuwen MA, van Rijswijk MH, Prevoo ML, Van 't Hof MA, van de Putte LB, et al. No increased mortality in patients with rheumatoid arthritis: up to 10 years of follow up from disease onset. Ann Rheum Dis 2000;59:954-8.

23. Radovits BJ, Fransen J, Al Shamma S, Eijsbouts AM, van Riel PL, Laan RF. Excess mortality emerges after 10 years in an inception cohort of early rheumatoid arthritis. Arthritis Care Res (Hoboken) 2010;62:362-70.

24. Wallberg-Jonsson S, Johansson H, Ohman M.L, Rantapaa-Dahlqvist S. Extent of inflammation predicts cardiovascular disease and overall mortality in seropositive rheumatoid arthritis. A retrospective cohort study from disease onset. J Rheumatol $1999 ; 26: 2562-71$.

25. Ropes MW, Bennett GA, Cobb S, Jacox R, Jessar RA. 1958 Revision of diagnostic criteria for rheumatoid arthritis. Bull Rheum Dis 1958;9:175-6.

26. Eberhardt KB, Rydgren LC, Pettersson H, Wollheim FA. Early rheumatoid arthritis onset, course, and outcome over 2 years. Rheumatol Int 1990;10:135-42.

27. Lindqvist E, Saxne T, Geborek P, Eberhardt K. Ten year outcome in a cohort of patients with early rheumatoid arthritis: health status, disease process, and damage. Ann Rheum Dis 2002;61:1055-9. 
28. van der Heijde DM, van 't Hof M, van Riel PL, van de Putte LB. Development of a disease activity score based on judgment in clinical practice by rheumatologists. J Rheumatol 1993;20:579-81.

29. Ekdahl C, Eberhardt K, Andersson SI, Svensson B. Assessing disability in patients with rheumatoid arthritis. Use of a Swedish version of the Stanford Health Assessment Questionnaire. Scand J Rheumatol 1988;17:263-71.

30. Larsen A, Dale K, Eek M. Radiographic evaluation of rheumatoid arthritis and related conditions by standard reference films. Acta Radiol Diagn (Stockh) 1977;18:481-91.

31. Lindqvist E, Eberhardt K, Klaus Bendtzen, Saxne T. Prognostic laboratory markers of radiographic outcome in rheumatoid arthritis. Ann Rheum Dis 2005;64:196-201.

32. Statistics Sweden. The Swedish Cause of Death Register (Statistiska centralbyrån. Dödsorsaker). Available online at: www.scb.se.

33. Kapetanovic MC, Lindqvist E, Simonsson M, Geborek P, Saxne T, Eberhardt K. Prevalence and predictive factors of comorbidity in rheumatoid arthritis patients monitored prospectively from disease onset up to 20 years: lack of association between inflammation and cardiovascular disease. Scand J Rheumatol. 2010;39 (5):353-9.

34. Emery P, Breedveld FC, Dougados M, Kalden JR, Schiff MH, Smolen JS. Early referral recommendation for newly diagnosed rheumatoid arthritis: evidence based development of a clinical guide. Ann Rheum Dis 2002;61:290-7.

35. Daviglus ML, Lloyd-Jones DM, Pirzada A. Preventing cardiovascular disease in the 21st century: therapeutic and preventive implications of current evidence. Am J Cardiovasc Drugs 2006;6:87-101.

36. Jansson SP, Andersson DK, Svärdsudd K. Mortality trends in subjects with and without diabetes during 33 years of follow-up. Diabetes Care 2010;33:551-6. 
37. Uhlig T, Heiberg T, Mowinckel P, Kvien TK. Rheumatoid arthritis is milder in the new millennium: health status in patients with rheumatoid arthritis 1994-2004. Ann Rheum Dis 2008;67:1710-15.

38. MacGregor AJ. Classification criteria for rheumatoid arthritis. Baillière's Clin Rheumatol 1995;9:287-304. 
Table 1. Baseline demographic and disease characteristics in the whole cohort, patients who died, and the remaining patients in the cohort.

\begin{tabular}{|c|c|c|c|c|}
\hline & $\begin{array}{l}\text { All patients (Lund } \\
\text { early RA cohort) } \\
(\mathrm{n}=183)\end{array}$ & $\begin{array}{l}\text { Patients who died } \\
\text { during the follow-up } \\
(\mathrm{n}=69)\end{array}$ & $\begin{array}{l}\text { Patients who are } \\
\text { still alive } \\
(\mathrm{n}=114)\end{array}$ & $\begin{array}{l}\text { Differences between patients } \\
\text { who died and those still alive } \\
\text { p-value }\end{array}$ \\
\hline Age (years) & $51.6(18-78 ; 12.4)$ & $60.1(36-78 ; 10.4)$ & $46.4(18-68 ; 10.6)$ & $0.000^{*}$ \\
\hline Gender (no. of females, \%) & $115(63)$ & $37(68)$ & $78(54)$ & $0.045 \dagger$ \\
\hline Duration of symptom at diagnosis (months) & $11(0-24 ; 6.9)$ & $11.6(0-24 ; 7.2)$ & $11.2(0-24)(6.6)$ & 0.921 \\
\hline Shared epitope (yes, \%) & 79 & 73 & 83 & 0.695 \\
\hline Shared epitope (double copies) (yes, \%) & 30 & 23 & 33 & 0.296 \\
\hline Smoking status at diagnosis (yes, \%) & 30 & 35 & 27 & $0.051 \dagger$ \\
\hline $\begin{array}{l}\text { Larsen score at diagnosis (0-200) } \\
\text { (hands and feet) }\end{array}$ & $8.5(0-41 ; 8.9)$ & $10.4(1-41 ; 9.7)$ & $7.3(0-36 ; 8.3)$ & $0.003^{*}$ \\
\hline RF-IgM positivity (yes, $\%$ ) & 76 & 71 & 79 & 0.552 \\
\hline Anti-CCP positivity (yes, \%) & 77 & 73 & 79 & 0.724 \\
\hline
\end{tabular}

Table 2. The impact of baseline demographic and disease characteristics on mortality risk.

\begin{tabular}{lllll}
\hline HAQ at diagnosis $(0-3)$ & $0.9(0-2.7 ; 0.6)$ & $1.0(0-2.7 ; 0.7)$ & $0.9(0-2.4 ; 0.6)$ & 0.390 \\
DAS at diagnosis $(0-10)$ & $3.3(1.2-6.2 ; 1.0)$ & $3.2(1.2-5.8 ; 1.1)$ & $3.3(1.6-6.2 ; 0.9)$ & 0.642 \\
ESR at diagnosis $(\mathrm{mm} / \mathrm{h})$ & $36.4(2-116 ; 27.8)$ & $40.2(2-116 ; 31.2)$ & $34.1(2-110 ; 25.2)$ & 0.302 \\
CRP at diagnosis $(\mathrm{mg} / \mathrm{L})$ & $26.6(0-199 ; 33.4)$ & $32.4(0-199 ; 40)$ & $23.4(0-133 ; 29.0)$ & 0.195 \\
NSAID at diagnosis (yes, \%) & 95 & 97 & 93 & 0.320 \\
Any co-morbidity at diagnosis, $\mathrm{n}(\%)$ & $78(43)$ & $36(52)$ & $42(37)$ & $0.030 \dagger$ \\
CVD at diagnosis, $\mathrm{n}(\%) \dot{5}$ & $18(10)$ & $13(19)$ & $5(4)$ & $0.001 \dagger$ \\
\hline
\end{tabular}

Figures for continuous variables given as mean (min-max; SD).

* Mann-Whitney U-test.

$\dagger \chi^{2}$ test.

$\ddagger$ CVD (excluding hypertension).

Table 3. The impact of baseline demographic and disease characteristics and treatment with DMARDs and steroids within a year after diagnosis on mortality risk. 


\begin{tabular}{lcccc}
\hline & p-value & HR & 95\% CI for HR & \\
\cline { 3 - 4 } & & & Lower & Upper \\
\hline Age at diagnosis (years) & 0.000 & 1.120 & 1.082 & 3.159 \\
Gender (male/female) & 0.023 & 1.932 & 1.095 & 3.813 \\
Smoking at diagnosis (yes) & 0.005 & 2.198 & 1.267 & 8.135 \\
CVD at diagnosis (yes/no) & 0.003 & 3.511 & 1.515 & 1.068 \\
HAQ at diagnosis (0-3) & 0.087 & 0.632 & 0.374 & 1.018 \\
ESR at diagnosis (mm/h) & 0.187 & 1.007 & 0.997 & 6.234 \\
Steroids at year 1 (yes/no) & 0.097 & 2.313 & 0.858 & 1.380 \\
DMARD at year 1 (yes/no) & 0.400 & 0.785 & 0.446 & \\
\hline
\end{tabular}

$\mathrm{HR}=$ Hazardratio

Figure 1. Long-term survival (95\% CI) among patients with RA followed prospectively since disease onset. Comparison with survival expected in Sweden for the same time period, adjusted for calendar year, age, and sex.

\section{Observed versus expected survival}

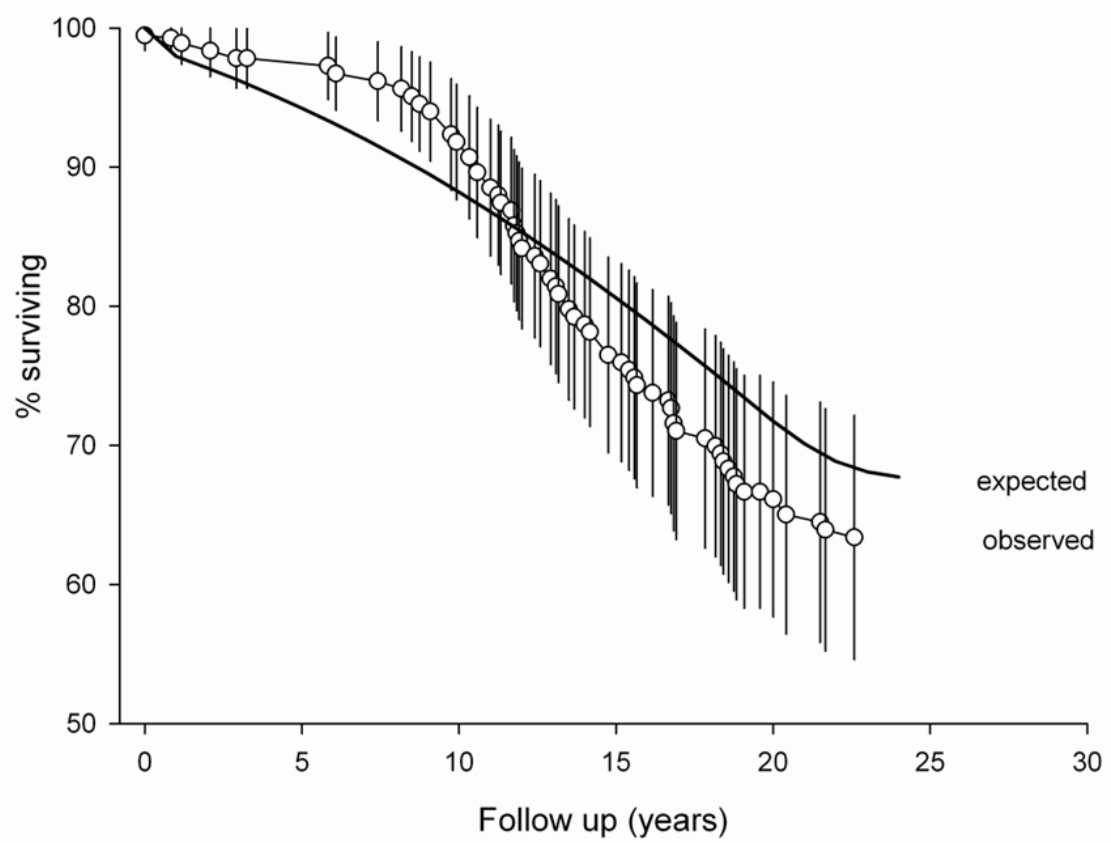

